\title{
Association of serum 25-hydroxyvitamin D and serum total cholesterol with depressive symptoms in Korean adults: the Fifth Korean National Health and Nutrition Examination Survey (KNHANES V, 2010-2012)
}

\author{
Soo-Hyun Lee ${ }^{1}$, Eunkyung Suh ${ }^{2}$, Kyung-Chae Park ${ }^{1, *}$, Ji-Hee Haam ${ }^{1}$, KyongChol Kim³ , \\ Hyung Suk Koo ${ }^{4}$, Beom-hee Choi ${ }^{5}$, Bo Youn Won ${ }^{1}$, Ki-Hyun Park ${ }^{1}$, Kye-Seon Park ${ }^{1}$, \\ Moon-Jong $\mathrm{Kim}^{1}$ and Young-Sang $\mathrm{Kim}^{1}$ \\ 'Department of Family Medicine, Bundang CHA Medical Center, CHA University, 59 Yatap-ro, Bundang-gu, \\ Seongnam-si, Gyeonggi-do 463-712, Republic of Korea: ${ }^{2}$ Department of Family Medicine, Chaum Hospital, CHA \\ University, Seoul, Republic of Korea: ${ }^{3}$ Antiaging Center, Chaum Life Center, CHA University, Seoul, Republic of \\ Korea: ${ }^{4}$ Bundang Oriental Hospital, Dongguk University, Kyeonggi-do, Republic of Korea: ${ }^{5} \mathrm{CHAUM}$ Medical Check- \\ UP Center, CHA University, Seoul, Republic of Korea
}

Submitted 29 February 2016: Accepted 22 May 2016: Accepted 24 May 2016: First published online 4 July 2016

\begin{abstract}
Objective: To examine the hypothesis that the association between vitamin D deficiency and depressive symptoms is dependent upon total cholesterol level in a representative national sample of the South Korean population.

Design: This was a population-based cross-sectional study.

Setting: The Fifth Korean National Health and Nutrition Examination Survey (KNHANES V, 2010-2012).

Subjects: We included 7198 adults aged $20-88$ years.

Results: The incidence of depressive symptoms in individuals with vitamin D deficiency (serum 25-hydroxyvitamin D $<20 \mathrm{ng} / \mathrm{ml}$ ) was 1.54-fold (95\% CI 1.20, 1.98) greater than in individuals without vitamin D deficiency (serum 25-hydroxyvitamin D $\geq 20 \mathrm{ng} / \mathrm{ml}$ ). The relationship was stronger in individuals with normal-to-borderline serum total cholesterol (serum total cholesterol $<240 \mathrm{mg} / \mathrm{dl}$; OR $=1.60 ; 95 \%$ CI 1.23 , $2 \cdot 08)$ and non-significant in individuals with high serum total cholesterol $(O R=0.97$; $95 \%$ CI $0.52,1 \cdot 81$ ) after adjustment for confounding variables (age, sex, BMI, alcohol consumption, smoking status, regular exercise, income level, education level, marital status, changes in body weight, perceived body shape, season of examination date and cholesterol profiles).

Conclusions: The association between vitamin D deficiency and depressive symptoms was weakened by high serum total cholesterol status. These findings suggest that both vitamin D and total cholesterol are important targets for the prevention and treatment of depression.
\end{abstract}

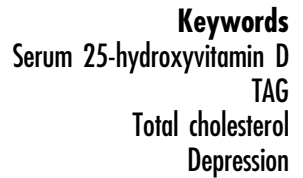

Vitamin D is involved in regulating serum concentrations of calcium and phosphate as well as bone mineralization ${ }^{(1-3)}$. Vitamin D is also involved in various pathophysiological processes in the human body; research has shown that vitamin D deficiency is a risk factor for CVD, cancer, diabetes mellitus, autoimmune disease and cognitive disorders $^{(4-6)}$. In addition, vitamin D supplementation has been reported to reduce the risk of these chronic diseases ${ }^{(7-9)}$. Therefore, vitamin D deficiency is an important and relevant health issue in modern society.

Depression is a disorder that has been correlated with vitamin D deficiency in several studies ${ }^{(10,11)}$.
The pathological mechanism explaining this phenomenon potentially involves the ability of vitamin D to cross the blood-brain barrier and bind to vitamin D receptors in the brain $^{(12,13)}$. Similarly, depression has also been related to serum cholesterol status ${ }^{(14,15)}$. Increasing evidence suggests that cholesterol affects cognitive function and mood status ${ }^{(16,17)}$. Low serum cholesterol has been identified as a risk factor for depression in some studies ${ }^{(14,18)}$; yet, other studies indicate an opposite (positive) correlation between serum cholesterol and depression ${ }^{(15)}$.

Vitamin D and cholesterol are not completely independent of one another. Several studies have demonstrated that 
high serum 25-hydroxyvitamin D (25(OH)D) is associated with a favourable serum cholesterol profile ${ }^{(19)}$. Research has also indicated a positive correlation between vitamin D and total cholesterol levels ${ }^{(19-22)}$; however, other studies have also suggested an opposite (negative) correlation between these variables ${ }^{(23)}$.

Clearly, there exists controversy over the relationships between vitamin D, cholesterol and depression; furthermore, no study to date has investigated correlations among these three factors simultaneously. Therefore, in the present study, we examined associations among vitamin D deficiency, serum cholesterol and depressive symptoms in a general population of South Korean adults using data from the Fifth Korean National Health and Nutritional Examination Survey (KNHANES V) conducted from 2010 to 2012. Our data characterize the relationship between serum $25(\mathrm{OH}) \mathrm{D}$ and depressive symptoms, as well as the interaction of this relationship with serum total cholesterol level.

\section{Methods}

\section{Study population}

The present study was performed on data from the KNHANES V, which was conducted by the Division of Chronic Disease Surveillance of the Korea Centers for Disease Control and Prevention between January 2010 and December 2012. The KNHANES is a survey for the assessment of health status, health behaviour, nutritional status and sociodemographic data in the civilian noninstitutionalized population of South Korea. A stratified, multistage probability sampling design was used for the selection of household units. Each year, 192 survey sections were randomly sampled and twenty households were selected from each section. Finally, 25534 individuals participated in the examination. For the purposes of our study, we selected individuals who were over the age of 20 years (Fig. 1). We excluded individuals who did not report essential variables, such as serum $25(\mathrm{OH}) \mathrm{D}$ concentration, serum cholesterol profiles or mental health questionnaire responses. We also excluded individuals with chronic diseases that might affect serum $25(\mathrm{OH}) \mathrm{D}$ concentration or mood status, including hypertension, diabetes mellitus, ischaemic heart disease, stroke, cancer, liver cirrhosis, renal failure, asthma, thyroid disease and arthritis. Additionally, we excluded females who were pregnant or lactating and individuals who were taking medications such as vitamins, hormones and statins. In total, 7198 individuals were included in our statistical analysis. The KNHANES was approved by the Institutional Review Board of the Centers for Disease Control and Prevention in South Korea. All participants who took part in the survey provided written informed consent.

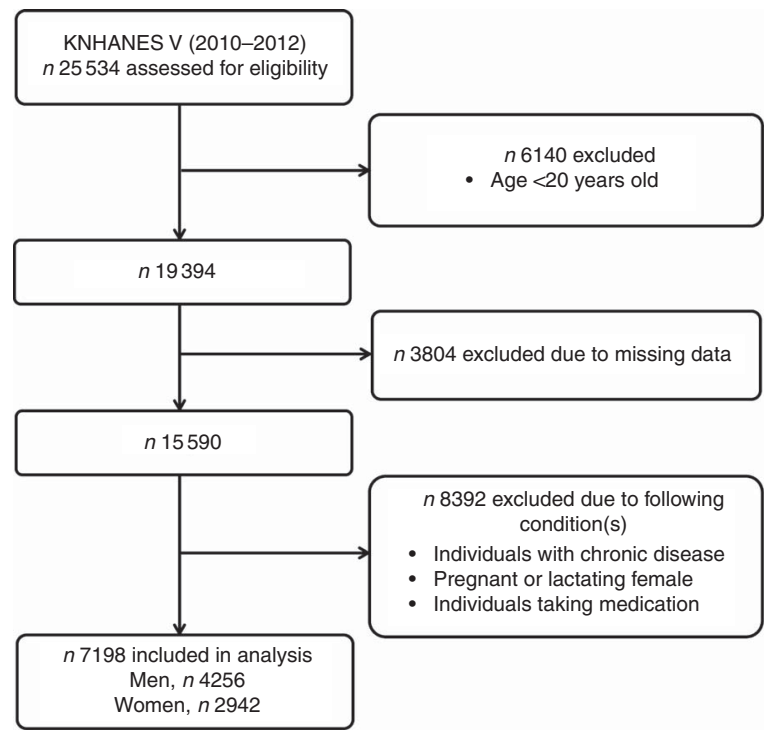

Fig. 1 Selection of the study population (KNHANES V, Fifth Korean National Health and Nutrition Examination Survey)

\section{General characteristics of participants}

Sex, age, BMI, marital status, income level, education level, alcohol consumption, smoking status, regular exercise, changes in body weight, perceived body shape and season were considered as confounding variables potentially affecting depression ${ }^{(24-27)}$. BMI $\left(\mathrm{kg} / \mathrm{m}^{2}\right)$ was defined as body weight divided by height squared. A standard mercury sphygmomanometer (Baumanometer; W.A. Baum Co., Inc., Copiague, NY, USA) was used on the right arm to measure blood pressure. Systolic blood pressure and diastolic blood pressure were measured with the participant in a seated position; measurements were taken twice with a 5 min interval and the values were averaged. Individuals who met the 7th Joint National Committee guideline criteria for hypertension (systolic blood pressure $\geq 140 \mathrm{mmHg}$ or diastolic blood pressure $\geq 90 \mathrm{mmHg}^{(28)}$ ) were classified as hypertensive and excluded from the analysis. Participants were divided into two groups according to marital status (single or married). Income level was divided into quartiles (lower, lower middle, upper middle and highest) based on questionnaire responses. Education level was classified according to four categories: graduation from elementary school, middle school, high school and university or higher. Participants were categorized as either non-smokers or current smokers. Alcohol consumption was evaluated using the Alcohol Use Disorders Identification Test (AUDIT) and individuals were divided into three groups according to the results: normal user ( $0-7$ points), hazardous user (8-15 points) and problematic user $(\geq 16 \text { points })^{(29)}$. Changes in body weight were classified according to three categories: weight gain, weight maintenance and weight loss. Perceived body shape was categorized as lean, normal or obese based on questionnaire responses. 
Regular exercise was defined as participation in a form of vigorous exercise (running, climbing, rapid cycling, rapid swimming, football, basketball, jumping rope, squash, singles tennis or carrying heavy objects) for $\geq 20$ min at least three times per week; moderate exercise (slow swimming, team tennis, volleyball or carrying light objects) for $\geq 30 \mathrm{~min}$ at least five times per week; or walking for $\geq 30$ min more than five times per week. The season of the examination date was classified as spring (March to May), summer (June to August), autumn (September to November), or winter (December to February).

\section{Vitamin $D$ and cholesterol levels measurement}

Fasting blood samples were collected for assessment. To evaluate vitamin D status, serum concentrations of 25(OH)D were measured by RIA (DiaSorin Inc., Stillwater, MN, USA) using a 1470 WIZARD gamma-counter (Perkin Elimer, Turku, Finland). Vitamin D status was categorized into two groups based on the American guideline ${ }^{(30)}$ : vitamin D deficiency $(25(\mathrm{OH}) \mathrm{D}<20 \mathrm{ng} / \mathrm{ml})$ and no deficiency $(25(\mathrm{OH}) \mathrm{D} \geq 20 \mathrm{ng} / \mathrm{ml})$. Total cholesterol, TAG and HDL cholesterol (HDL-C) were measured by an enzymatic technique using a Hitachi Automatic Analyser 7600 (Hitachi, Tokyo, Japan). Because LDL cholesterol data were not available for all participants in the KNHANES V, we excluded LDL cholesterol levels from our analysis. Total cholesterol level was categorized into three groups: desirable $(<200 \mathrm{mg} / \mathrm{dl})$, borderline high $(200-239 \mathrm{mg} / \mathrm{dl})$ and high $(\geq 240 \mathrm{mg} / \mathrm{dl})$. HDL-C level was categorized into two groups: low $(<40 \mathrm{mg} / \mathrm{dl})$ and normal $(\geq 40 \mathrm{mg} / \mathrm{dl})$. TAG level was also categorized into two groups: high $(\geq 200 \mathrm{mg} / \mathrm{dl})$ and normal to borderline $(<200 \mathrm{mg} / \mathrm{dl})$. Cholesterol classifications were based on the National Cholesterol Education Program (NCEP) Adult Treatment Panel III (ATP III) guideline ${ }^{(31)}$.

\section{Depressive symptoms assessment}

Depressive symptoms were assessed by self-reporting. Participants were asked whether they had experienced feelings of sadness or despair that interfered with everyday life and persisted for longer than 2 weeks in the past year. Those who answered 'yes' were classified as exhibiting depressive symptoms ${ }^{(32,33)}$.

\section{Statistical analysis}

All statistical analyses were performed using the statistical software package PASW Statistics Version 18.0. $P$ values of $<0.05$ were considered to be statistically significant. Clinical and demographic characteristics were analysed according to the presence or absence of self-reported depressive symptoms. Continuous variables were analysed using $t$ tests and nominal variables were analysed using $\chi^{2}$ tests. Associations between serum 25(OH)D and depressive symptoms were analysed using multiple logistic regressions. Odds ratios and 95\% confidence intervals were compared to assess changes in the association between serum $25(\mathrm{OH}) \mathrm{D}$ and depressive symptoms according to total cholesterol level. Confounding factors potentially affecting serum $25(\mathrm{OH}) \mathrm{D}$ and depressive symptoms were defined previously ${ }^{(18,34-39)}$. Age, sex, BMI, marital status, income level, education level, alcohol consumption, smoking status, regular exercise, changes in body weight, perceived body shape, season of the examination date, total cholesterol, HDL-C and TAG were adjusted for in the logistic regression analysis. To assess associations between different cholesterol profiles and depressive symptoms, odds ratios and 95\% confidence intervals of each cholesterol profile for depressive symptoms were calculated using multiple logistic regressions. We classified cholesterol profiles into two groups based on the NCEP ATP III guideline ${ }^{(31)}$ : total cholesterol level and TAG level were divided into high and normal-toborderline groups, and HDL-C level was divided into low and normal groups. We adjusted for the same confounding factors as in the analysis of serum 25(OH)D and depressive symptoms except for cholesterol profile. A correlation between serum $25(\mathrm{OH}) \mathrm{D}$ and total cholesterol level was analysed using a multiple linear regression analysis adjusted for age, sex, BMI, income level, education level, alcohol consumption, smoking status, regular exercise and changes in body weight, as these factors have been correlated with serum lipid concentrations in previous studies ${ }^{(40-43)}$. Changes in the correlation between serum 25(OH)D and total cholesterol level were analysed by examining the strength of this correlation in the normalto-borderline total cholesterol group and the high total cholesterol group.

\section{Results}

\section{Characteristics of study participants}

Study participants' characteristics are summarized according to the presence or absence of depressive symptoms in Table 1 . The depressive group consisted of 695 individuals (9.7\%). The mean age of each group (depressive and non-depressive) was approximately 39 years (range 20-88 years). There was a higher proportion of women in the depressive group relative to the nondepressive group. Moreover, income and education levels were lower in the depressive group than in the nondepressive group. Problematic alcohol consumption and changes in weight were more prevalent whereas regular exercise was lower in the depressive group relative to the non-depressive group. No seasonal variation in the reporting of depressive symptoms was noted.

With regard to biochemical characteristics, serum $25(\mathrm{OH}) \mathrm{D}$ was lower and the prevalence of vitamin $\mathrm{D}$ deficiency was higher in the depressive group than in the non-depressive group. Moreover, HDL-C levels were 
Table 1 Study participants' characteristics according to the presence or absence of depressive symptoms ${ }^{*}$; adults $(n$ 7198) aged 20-88 years, Fifth Korean National Health and Nutrition Examination Survey (KNHANES V, 2010-2012)

\begin{tabular}{|c|c|c|c|c|c|}
\hline \multirow[b]{2}{*}{ Characteristic } & \multicolumn{2}{|c|}{$\begin{array}{l}\text { Individuals without depressive symptoms } \dagger \\
\qquad(n \text { 6503) }\end{array}$} & \multicolumn{2}{|c|}{$\begin{array}{l}\text { Individuals with depressive symptoms } \\
\qquad(n \text { 695) }\end{array}$} & \multirow[b]{2}{*}{$P$ valuef } \\
\hline & Mean or \% & SD & Mean or $\%$ & SD & \\
\hline Age (years), mean and SD & 39.04 & $13 \cdot 16$ & 39.23 & 13.46 & 0.739 \\
\hline Sex, female (\%) & 39.3 & - & $55 \cdot 5$ & - & $<0.001$ \\
\hline BMI $\left(\mathrm{kg} / \mathrm{m}^{2}\right)$, mean and SD & 23.52 & $3 \cdot 31$ & 23.21 & 3.46 & 0.099 \\
\hline Marital status, married (\%) & 30.5 & - & 31.6 & - & 0.648 \\
\hline Income level (\%) & & & & & 0.004 \\
\hline Lowest & $25 \cdot 5$ & - & 33.2 & - & \\
\hline Lower middle & $25 \cdot 5$ & - & 24.5 & - & \\
\hline Upper middle & $25 \cdot 3$ & - & $22 \cdot 2$ & - & \\
\hline Highest & 23.7 & - & $20 \cdot 1$ & - & \\
\hline Education level (\%) & & & & & 0.002 \\
\hline Elementary school & $6 \cdot 7$ & - & $10 \cdot 6$ & - & \\
\hline Middle school & 6.9 & - & $8 \cdot 1$ & - & \\
\hline High school & 43.7 & - & $45 \cdot 6$ & - & \\
\hline University & $42 \cdot 8$ & - & 35.7 & - & \\
\hline Smoking status, current smoker (\%) & $32 \cdot 9$ & - & 34.7 & - & 0.423 \\
\hline Alcohol consumption (\%) & & & & & $<0.001$ \\
\hline Normal user & 58.4 & - & $54 \cdot 2$ & _- & \\
\hline Hazardous user & $27 \cdot 1$ & - & $22 \cdot 8$ & - & \\
\hline Problematic user & $14 \cdot 4$ & - & 23.0 & - & \\
\hline Regular exercise§ (\%) & $50 \cdot 7$ & - & 43.6 & - & 0.004 \\
\hline Perceived body shape (\%) & & & & & 0.278 \\
\hline Lean & $17 \cdot 8$ & _- & 18.6 & _ & \\
\hline Normal & $40 \cdot 2$ & - & $36 \cdot 4$ & - & \\
\hline Obese & 41.9 & _- & $45 \cdot 0$ & _- & \\
\hline Changes in body weight (\%) & & & & & $<0.001$ \\
\hline Weight loss & $12 \cdot 5$ & - & $17 \cdot 4$ & - & \\
\hline Maintenance & $64 \cdot 1$ & - & $54 \cdot 6$ & - & \\
\hline Weight gain & $23 \cdot 4$ & - & 28.1 & - & \\
\hline Season $(\%)$ & & & & & $0 \cdot 187$ \\
\hline Spring & 23.4 & - & $26 \cdot 9$ & - & \\
\hline Summer & $27 \cdot 3$ & - & $27 \cdot 0$ & - & \\
\hline Autumn & $25 \cdot 0$ & - & 21.4 & - & \\
\hline Winter & $24 \cdot 3$ & - & $24 \cdot 8$ & - & \\
\hline $25(\mathrm{OH}) \mathrm{D}(\mathrm{ng} / \mathrm{ml})$, mean and SD & $17 \cdot 14$ & 5.76 & $15 \cdot 80$ & $5 \cdot 84$ & $<0.001$ \\
\hline Vitamin D deficiency (\%) & $70 \cdot 7$ & - & $80 \cdot 3$ & - & $<0.001$ \\
\hline Total cholesterol $(\mathrm{mg} / \mathrm{dl})$, mean and SD & $187 \cdot 10$ & 34.92 & $188 \cdot 18$ & 37.97 & 0.562 \\
\hline TAG (mg/dl), mean and SD & 130.42 & 90.51 & 134.53 & 93.72 & 0.586 \\
\hline $\mathrm{HDL}-\mathrm{C}(\mathrm{mg} / \mathrm{dl})$ mean and SD & 52.71 & 12.50 & 54.52 & 13.59 & 0.009 \\
\hline
\end{tabular}

25(OH)D, serum 25-hydroxyvitamin D; HDL-C, HDL cholesterol.

*Values are presented as means and standard deviations or as percentages.

†Depressive symptoms were defined as feelings of sadness or despair that interfered with everyday life and persisted for longer than 2 weeks in the previous year.

$\ddagger$ The $t$ test was used to analyse continuous variables and the $x^{2}$ test was used to analyse categorical variables.

§Regular exercise was defined as vigorous physical activity lasting $\geq 20 \mathrm{~min}$ at least three times per week, moderate physical activity lasting $\geq 30$ min at least five times per week, or walking for $\geq 30$ min more than five times per week.

higher in the depressive group than in the non-depressive group. There were no significant differences in total cholesterol or TAG levels between the two groups.

\section{Association between serum 25-hydroxyvitamin D and depressive symptoms, and the interaction of this association with total cholesterol level}

We first analysed the association between vitamin D deficiency and depressive symptoms using a multiple logistic regression analysis. Odds ratios and 95\% confidence intervals are presented in Table 2. First, we analysed all participants independent of total cholesterol level. In the unadjusted model, there was a positive association between vitamin D deficiency and depressive symptoms (OR $=1 \cdot 69 ; 95 \%$ CI $1 \cdot 35,2 \cdot 11$ ) and this positive association was preserved after adjusting for age, sex, BMI, alcohol consumption, smoking status, regular exercise, income level, education level, marital status, changes in body weight, perceived body shape, season and cholesterol profiles (OR $=1.54 ; 95 \%$ CI 1.20, 1.98). After dividing participants according to total cholesterol level, the positive association changed: the number of individuals with normal-to-borderline total cholesterol was 6673 while the number with high total cholesterol was 525. In the normalto-borderline total cholesterol group (total cholesterol $<240 \mathrm{mg} / \mathrm{dl}$ ), the positive association between vitamin D deficiency and depressive symptoms was preserved 
Table 2 Association between serum 25-hydroxyvitamin D (25(OH)D) and depressive symptoms according to serum total cholesterol (TC) level among adults $(n$ 7198) aged 20-88 years, Fifth Korean National Health and Nutrition Examination Survey (KNHANES V, 2010-2012)

\begin{tabular}{|c|c|c|c|c|c|c|c|}
\hline \multirow[b]{3}{*}{ Category } & \multirow[b]{3}{*}{$25(\mathrm{OH}) \mathrm{D}(\mathrm{ng} / \mathrm{ml})$} & \multicolumn{6}{|c|}{ Depressive symptoms } \\
\hline & & \multicolumn{3}{|c|}{ Model $1^{*}$} & \multicolumn{3}{|c|}{ Model $2 \dagger$} \\
\hline & & OR & $95 \% \mathrm{Cl}$ & $P$ value & OR & $95 \% \mathrm{Cl}$ & $P$ value $\neq$ \\
\hline All participants ( $n$ 7198) & $\begin{array}{l}<20 \\
\geq 20 \S\end{array}$ & 1.69 & $\begin{array}{l}1.35,2.11 \\
1.00\end{array}$ & $<0.001$ & 1.54 & $\begin{array}{l}1.20,1.98 \\
1.00\end{array}$ & $<0.001$ \\
\hline $\begin{array}{l}\mathrm{TC}<240 \mathrm{mg} / \mathrm{dl} \text { group } \\
(n 6673)\end{array}$ & $\begin{array}{l}<20 \\
\geq 20 \S\end{array}$ & 1.75 & $\begin{array}{l}1.38,2.23 \\
1.00\end{array}$ & $<0.001$ & $1 \cdot 60$ & $\begin{array}{l}1.23,2.08 \\
1.00\end{array}$ & 0.001 \\
\hline $\begin{array}{l}\mathrm{TC} \geq 240 \mathrm{mg} / \mathrm{dl} \text { group } \\
(n 525)\end{array}$ & $\begin{array}{l}<20 \\
\geq 20 \S\end{array}$ & $1 \cdot 29$ & $\begin{array}{l}0.69,2.44 \\
1.00\end{array}$ & 0.426 & 0.97 & $\begin{array}{l}0.52,1.81 \\
1.00\end{array}$ & 0.927 \\
\hline
\end{tabular}

*Unadjusted.

†Adjusted for age, sex, BMI, alcohol consumption, smoking status, regular exercise, income level, education level, marital status, changes in body weight, perceived body shape, season of the examination date and cholesterol profiles.

$\ddagger$ A multiple logistic regression model was used.

§Reference group.

Table 3 Associations between serum cholesterol profiles and depressive symptoms among adults ( $n$ 7198) aged 20-88 years, Fifth Korean National Health and Nutrition Examination Survey (KNHANES V, 2010-2012)

\begin{tabular}{|c|c|c|c|c|c|c|}
\hline \multirow[b]{3}{*}{ Cholesterol profile } & \multicolumn{6}{|c|}{ Depressive symptoms } \\
\hline & \multicolumn{3}{|c|}{ Model $1^{*}$} & \multicolumn{3}{|c|}{ Model $2 \dagger$} \\
\hline & OR & $95 \% \mathrm{Cl}$ & $P$ value & OR & $95 \% \mathrm{Cl}$ & $P$ value \\
\hline \multicolumn{7}{|c|}{ Total cholesterol (mg/dl) } \\
\hline $\begin{aligned} & \geq 240 \\
&<240 \S \\
& \text { TAG }(\mathrm{mg} / \mathrm{dl})\end{aligned}$ & 1.32 & $1.00^{0.93,1.85}$ & 0.117 & 1.30 & $1.00^{0.91,1.85}$ & 0.146 \\
\hline $\begin{array}{l}\geq 200 \\
<200 \S\end{array}$ & 0.97 & $1.00^{0.72,1.29}$ & 0.557 & 1.04 & $1.00^{0.77,1.43}$ & 0.785 \\
\hline \multicolumn{7}{|l|}{ HDL-C (mg/dl) } \\
\hline $\begin{array}{l}<40 \\
\geq 40 \S\end{array}$ & 0.87 & $1.00^{0.65,1.16}$ & 0.350 & $1 \cdot 14$ & $1.00^{0.83,1.55}$ & 0.423 \\
\hline
\end{tabular}

HDL-C, HDL cholesterol.

*Unadjusted.

†Adjusted for age, sex, BMI, alcohol consumption, smoking status, regular exercise, income level, education level, marital status, changes in body weight, perceived body shape and season of the examination date.

$\ddagger$ A multiple logistic regression model was used.

$\S$ Reference group.

(Model 1: OR $=1 \cdot 75 ; 95 \%$ CI $1 \cdot 38,2 \cdot 23$, Model 2: $\mathrm{OR}=1.60 ; 95 \% \mathrm{CI} 1 \cdot 23,2 \cdot 08)$; however, this association became non-significant in the high total cholesterol group (Model 1: OR $=1 \cdot 29$; $95 \%$ CI 0.69, 2.44, Model 2: $\mathrm{OR}=0.97 ; 95 \% \mathrm{CI} 0.52,1.81$ ). No changes in the association between vitamin $\mathrm{D}$ deficiency and depressive symptoms were observed when participants were divided according to HDL-C or TAG level.

\section{Association between cholesterol profiles and depressive symptoms}

We evaluated the association between cholesterol profiles and the presence of depressive symptoms using a multiple logistic regression analysis. The odds ratios and 95\% confidence intervals are presented in Table 3. No significant associations between cholesterol profiles and depressive symptoms were observed even after adjusting for confounding factors.

\section{Correlation between serum 25-bydroxyvitamin $D$ and total cholesterol level}

We evaluated the association between serum 25(OH)D and total cholesterol level using a multiple linear regression analysis. We also analysed the change of correlation between serum 25(OH)D and total cholesterol level according to total cholesterol status. The regression coefficients $(\beta)$ between serum 25(OH)D and total cholesterol are presented in Table 4 . In the unadjusted model, significant positive correlations were observed between serum 25(OH)D and total cholesterol for all participants $(\beta=0.481, P<0.001)$ and in the normal-to-borderline total cholesterol group $(\beta=0.413, P<0.001)$. However, these 
Table 4 Correlation between serum 25-hydroxyvitamin D (25(OH)D) and serum total cholesterol (TC) level according to total cholesterol status among adults $(n$ 7198) aged $20-88$ years, Fifth Korean National Health and Nutrition Examination Survey (KNHANES V, 2010-2012)

\begin{tabular}{|c|c|c|c|c|}
\hline \multirow[b]{2}{*}{ Category } & \multicolumn{2}{|c|}{ Model $1^{*}$} & \multicolumn{2}{|c|}{ Model 2† } \\
\hline & $\beta$ & $P$ value & $\beta$ & $P$ value \\
\hline All participants ( $n$ 7198) & 0.481 & $<0.001$ & 0.034 & 0.714 \\
\hline $\begin{array}{c}\mathrm{TC}<240 \mathrm{mg} / \mathrm{dl} \text { group } \\
(n 6673)\end{array}$ & 0.413 & $<0.001$ & 0.080 & $0 \cdot 288$ \\
\hline $\begin{array}{l}\mathrm{TC} \geq 240 \mathrm{mg} / \mathrm{dl} \text { group } \\
(n 525)\end{array}$ & -0.408 & 0.058 & -0.480 & 0.038 \\
\hline
\end{tabular}

$\beta$, regression coefficient.

*Unadjusted.

†Adjusted for age, sex, BMI, alcohol consumption, smoking status, regular exercise, income level, education level and changes in body weight. $\ddagger$ A multiple linear regression model was used.

correlations became non-significant after adjusting for age, sex, BMI, alcohol consumption, smoking status, regular exercise, income, education and changes in body weight ( $\beta=0.034, P=0.714 v \cdot \beta=0.080, P=0 \cdot 288$ ). Alternatively, there was a significant negative correlation between serum $25(\mathrm{OH}) \mathrm{D}$ and total cholesterol level in the high total cholesterol group that was preserved even after adjustment for the abovementioned factors $(\beta=-0.480$, $P=0 \cdot 038)$.

\section{Discussion}

In the current population-based cross-sectional study, we confirmed the presence of an association between vitamin D deficiency and depressive symptoms in a population of South Korean adults after controlling for confounding variables. Vitamin D-deficient persons with normal-to-borderline serum total cholesterol had an approximately 1.6-fold increased risk of depressive symptoms. Furthermore, we demonstrated that this association was absent in a sub-population of individuals with high serum total cholesterol. These data suggest that individuals with high serum total cholesterol may be less susceptible to the effects of vitamin D deficiency on mood status and particularly depression. Previous studies that have investigated serum $25(\mathrm{OH}) \mathrm{D}$ or total cholesterol in the context of depression have focused on only one factor and yielded inconsistent results ${ }^{(10,11,44)}$. These previous studies were limited by small sample sizes and confounding socio-economic and demographic factors. The population-based nature of our study and our consideration of a variety of confounding factors related to serum $25(\mathrm{OH}) \mathrm{D}$, cholesterol status and depression therefore bolster the strength of our findings.

The underlying pathophysiology of depression remains unclear; however, several potential mechanisms have been put forth. Vitamin $\mathrm{D}$ receptors are abundant in many brain areas including the cingulate cortex and hippocampus. Vitamin D plays a role in neuroimmunomodulation, the regulation of neurotrophic factors, neuroprotection, neuroplasticity and brain development ${ }^{(12,13)}$. To this end, vitamin $\mathrm{D}$ has been hypothesized to play a direct role in the aetiology of depression.

Many studies have examined the relationship between serum cholesterol and depression. In some studies, individuals with depressive symptoms were noted to have lower serum total cholesterol ${ }^{(14,15)}$. One study suggested that lower total cholesterol might produce depression by altering the micro-viscosity of plasma membranes as a mechanism to decrease central serotonin transmission ${ }^{(45)}$. Alternatively, other studies have suggested that depression decreases serum total cholesterol by suppressing appetite and inhibiting cholesterol synthesis ${ }^{(16,17)}$. Dysregulation of the hypothalamic-pituitary-adrenal axis observed in depression can also alter cholesterol metabolism ${ }^{(46)}$. In the present study we failed to observe any relationship between serum total cholesterol and the presence of depressive symptoms.

It is known that both vitamin D and total cholesterol are factors relevant to depression; however, the correlation between these factors has been reported inconsistently in previous studies. While some studies reported a negative correlation $^{(23,47,48)}$, others indicated a positive correlation between vitamin D and total cholesterol ${ }^{(19-22)}$. Vitamin D can influence serum cholesterol levels via a direct effect on adipogenesis ${ }^{(49,50)}$. Indirectly, vitamin D can increase intestinal calcium absorption and thus fat absorption ${ }^{(51,52)}$, which has reciprocal effects on serum parathyroid hormone and lipolysis ${ }^{(53)}$. Baseline total cholesterol level has also been identified as a determinant of $25(\mathrm{OH}) \mathrm{D}$ synthesis after UV-B exposure ${ }^{(54)}$. In our study, vitamin D and total cholesterol were negatively correlated only in individuals with high total cholesterol status. The reason for this observation is unclear; however, it may partly explain the inconsistent results of previous studies investigating the relationship between vitamin $\mathrm{D}$ and serum total cholesterol.

Although we did not observe a significant association between total cholesterol and depression symptoms in the present study, a recent meta-analysis confirmed the inverse association of total cholesterol with depression in a total of thirty previous studies ${ }^{(18)}$. Thus, we can suppose that both vitamin D and total cholesterol are independent factors related to depression in individuals with normal-toborderline total cholesterol. However, our data suggest that in individuals with high total cholesterol as classified by the NCEP ATP III guideline, vitamin D and total cholesterol influence one another in a manner that alters their correlations with depression.

There were several limitations in the present study. First, the cross-sectional design of the study prohibited us from making conclusions regarding the causality of relationships among vitamin D deficiency, total cholesterol and 
depressive symptoms. The directionality of associations between these factors also remains unclear. A second limitation is related to the definition of depressive symptoms in our study. Individuals were included in the depressive group if they answered 'yes' to a questionnaire regarding the experience of depressive symptoms for a period of at least 2 weeks in the previous year. While previous studies have shown the validity of singlequestion screening for depression ${ }^{(33,55)}$, there still exists some controversy concerning this method ${ }^{(56)}$. Future studies should validate our findings through the use of wellaccepted scoring systems for the diagnosis of depression. Third, other potential confounding factors affecting depressive symptoms, vitamin D and total cholesterol may have been overlooked in our study. Future analyses should consider information about dietary intake (energy and saturated fat intakes) and menopause status.

To the best of our knowledge, the present study is the first to examine the association of vitamin $\mathrm{D}$ deficiency and depressive symptoms according to serum total cholesterol status. As aforementioned, it is likely that the presence of vitamin D receptors in the brain contributes to the pathophysiology of depression and that total cholesterol can influence these processes. However, it was beyond the scope of our study to identify the definite mechanisms explaining our result. Nevertheless, our results have important implications for the use of vitamin D supplementation in vitamin D-deficient individuals with depression. In conclusion, our data indicate that high serum total cholesterol weakens the association between vitamin D deficiency and depressive symptoms in adults.

\section{Acknowledgements}

Acknowledgements: The authors are thankful to the Division of Chronic Disease Surveillance, Korea Centers for Disease Control and Prevention for providing the KNHANES V data. Financial support: This research received no specific grant from any funding agency in the public, commercial or not-for-profit sectors. Conflict of interest: The authors declare no conflict of interest relevant to this article. Authorship: S.-H.L. drafted the article. K.-C.P. conceived and designed the study and takes full responsibility for the work as a whole. E.S., J.-H.H., K.K. and H.S.K. contributed to data interpretation and gave valuable suggestions and feedback. B.Y.W., K.-H.P., S.-H.L. and K.-S.P. analysed the data. M.-J.K., Y.-S.K. and B.C. revised the article critically for important intellectual content. Etbics of buman subject participation: This study was conducted according to the guidelines laid down in the Declaration of Helsinki and all procedures involving human subjects were approved by the Institutional Review Board of the Centers for Disease Control and Prevention in South Korea. Written informed consent was obtained from all subjects.

\section{References}

1. Dawson-Hughes B (2004) Racial/ethnic considerations in making recommendations for vitamin $\mathrm{D}$ for adult and elderly men and women. Am J Clin Nutr 80, 6 Suppl., 1763S-1766S.

2. DeLuca HF (2004) Overview of general physiologic features and functions of vitamin D. Am J Clin Nutr 80, 6 Suppl., 1689S-1696S.

3. Khazai N, Judd SE \& Tangpricha V (2008) Calcium and vitamin D: skeletal and extraskeletal health. Curr Rheumatol Rep 10, 110-117.

4. Holick MF (2004) Vitamin D: importance in the prevention of cancers, type 1 diabetes, heart disease, and osteoporosis. Am J Clin Nutr 79, 362-371.

5. Holick MF (2007) Vitamin D deficiency. N Engl J Med 357, 266-281.

6. Przybelski RJ \& Binkley NC (2007) Is vitamin D important for preserving cognition? A positive correlation of serum 25-hydroxyvitamin D concentration with cognitive function. Arch Biochem Biophys 460, 202-205.

7. Teng M, Wolf M, Ofsthun MN et al. (2005) Activated injectable vitamin D and hemodialysis survival: a historical cohort study. J Am Soc Nephrol 16, 1115-1125.

8. Shoji T, Shinohara K, Kimoto E et al. (2004) Lower risk for cardiovascular mortality in oral $1 \alpha$-hydroxy vitamin $\mathrm{D}_{3}$ users in a haemodialysis population. Nephrol Dial Transplant 19, 179-184.

9. Vacek JL, Vanga SR, Good M et al. (2012) Vitamin D deficiency and supplementation and relation to cardiovascular health. Am J Cardiol 109, 359-363.

10. Wilkins CH, Sheline YI, Roe CM et al. (2006) Vitamin D deficiency is associated with low mood and worse cognitive performance in older adults. Am J Geriatr Psychiatry 14, 1032-1040.

11. May HT, Bair TL, Lappe DL et al. (2010) Association of vitamin D levels with incident depression among a general cardiovascular population. Am Heart J 159, 1037-1043.

12. Anglin RE, Samaan Z, Walter SD et al. (2013) Vitamin D deficiency and depression in adults: systematic review and meta-analysis. Br J Psychiatry 202, 100-107.

13. Fernandes de Abreu DA, Eyles D \& Feron F (2009) Vitamin D, a neuro-immunomodulator: implications for neurodegenerative and autoimmune diseases. Psychoneuroendocrinology 34, Suppl. 1, S265-S277.

14. Tedders SH, Fokong KD, McKenzie LE et al. (2011) Low cholesterol is associated with depression among US household population. J Affect Disord 135, 115-121.

15. Manfredini R, Caracciolo S, Salmi R et al. (2000) The association of low serum cholesterol with depression and suicidal behaviours: new hypotheses for the missing link. J Int Med Res 28, 247-257.

16. Terao T, Nakamura J, Yoshimura R et al. (2000) Relationship between serum cholesterol levels and meta-chlorophenylpiperazine-induced cortisol responses in healthy men and women. Psychiatry Res 96, 167-173.

17. Feingold KR \& Grunfeld C (1992) Role of cytokines in inducing hyperlipidemia. Diabetes 41, Suppl. 2, 97-101.

18. Shin JY, Suls J \& Martin R (2008) Are cholesterol and depression inversely related? A meta-analysis of the association between two cardiac risk factors. Ann Behav Med 36, 33-43.

19. Jorde R, Figenschau Y, Hutchinson M et al. (2010) High serum 25-hydroxyvitamin D concentrations are associated with a favorable serum lipid profile. Eur J Clin Nutr 64, $1457-1464$.

20. Kumar J, Muntner P, Kaskel FJ et al. (2009) Prevalence and associations of 25-hydroxyvitamin $\mathrm{D}$ deficiency in US children: NHANES 2001-2004. Pediatrics 124, e362-e370. 
21. Giovannucci E, Liu Y, Hollis BW et al. (2008) 25-Hydroxyvitamin D and risk of myocardial infarction in men: a prospective study. Arch Intern Med 168, 1174-1180.

22. Bolland MJ, Bacon CJ, Horne AM et al. (2010) Vitamin D insufficiency and health outcomes over $5 \mathrm{y}$ in older women. Am J Clin Nutr 91, 82-89.

23. Melamed ML, Michos ED, Post W et al. (2008) 25-Hydroxyvitamin D levels and the risk of mortality in the general population. Arch Intern Med 168, 1629-1637.

24. Dixon JB, Dixon ME \& O'Brien PE (2003) Depression in association with severe obesity: changes with weight loss. Arch Intern Med 163, 2058-2065.

25. Kim O \& Kim K (2001) Body weight, self-esteem, and depression in Korean female. Adolescence 36, 315-322.

26. Strohle A (2009) Physical activity, exercise, depression and anxiety disorders. J Neural Transm (Vienna) 116, 777-784.

27. Blazer DG, Kessler RC, McGonagle KA et al. (1994) The prevalence and distribution of major depression in a national community sample: the National Comorbidity Survey. Am J Psychiatry 151, 979-986.

28. Chobanian AV, Bakris GL, Black HR et al. (2003) Seventh report of the Joint National Committee on Prevention, Detection, Evaluation, and Treatment of High Blood Pressure. Hypertension 42, 1206-1252.

29. Saunders JB, Aasland OG, Babor TF et al. (1993) Development of the Alcohol Use Disorders Identification Test (AUDIT): WHO Collaborative Project on Early Detection of Persons with Harmful Alcohol Consumption - II. Addiction 88, 791-804.

30. Dawson-Hughes B, Heaney RP, Holick MF et al. (2005) Estimates of optimal vitamin D status. Osteoporos Int 16, 713-716.

31. National Cholesterol Education Program Expert Panel on Detection, Evaluation, and Treatment of High Blood Cholesterol in Adults (Adult Treatment Panel III) (2002) Third Report of the National Cholesterol Education Program (NCEP) Expert Panel on Detection, Evaluation, and Treatment of High Blood Cholesterol in Adults (Adult Treatment Panel III) final report. Circulation 106, 3143-3421.

32. Watkins C, Daniels L, Jack C et al. (2001) Accuracy of a single question in screening for depression in a cohort of patients after stroke: comparative study. BMJ 323, 1159.

33. Le Strat Y \& Dubertret C (2013) A single question to screen for major depression in the general population. Compr Psychiatry 54, 831-834.

34. Rhee SY, Hwang YC, Chung HY et al. (2012) Vitamin D and diabetes in Koreans: analyses based on the Fourth Korea National Health and Nutrition Examination Survey (KNHANES), 2008-2009. Diabetic Med 29, 1003-1010.

35. Saliba W, Barnett-Griness O \& Rennert G (2013) The relationship between obesity and the increase in serum $25(\mathrm{OH})$ $\mathrm{D}$ levels in response to vitamin $\mathrm{D}$ supplementation. Osteoporos Int 24, 1447-1454.

36. Cutillas-Marco E, Fuertes-Prosper A, Grant WB et al. (2012) Vitamin D deficiency in South Europe: effect of smoking and aging. Photodermatol Photoimmunol Photomed 28, $159-161$.

37. Lorant V, Deliege D, Eaton W et al. (2003) Socioeconomic inequalities in depression: a meta-analysis. Am J Epidemiol 157, 98-112.

38. Thuesen B, Husemoen L, Fenger M et al. (2012) Determinants of vitamin D status in a general population of Danish adults. Bone 50, 605-610.
39. Alvisa-Negrin J, Gonzalez-Reimers E, Santolaria-Fernandez F et al. (2009) Osteopenia in alcoholics: effect of alcohol abstinence. Alcohol Alcohol 44, 468-475.

40. Wamala SP, Wolk A, Schenck-Gustafsson K et al. (1997) Lipid profile and socioeconomic status in healthy middle aged women in Sweden. J Epidemiol Community Health 51, 400-407.

41. Whitehead TP, Robinson D \& Allaway SL (1996) The effects of cigarette smoking and alcohol consumption on blood lipids: a dose-related study on men. Ann Clin Biochem 33, 99-106.

42. Leenen R, van der Kooy K, Meyboom S et al. (1993) Relative effects of weight loss and dietary fat modification on serum lipid levels in the dietary treatment of obesity. J Lipid Res $\mathbf{3 4}$, 2183-2191.

43. Monda KL, Ballantyne CM \& North KE (2009) Longitudinal impact of physical activity on lipid profiles in middle-aged adults: the Atherosclerosis Risk in Communities Study. J Lipid Res 50, 1685-1691.

44. Pan A, Lu L, Franco OH et al. (2009) Association between depressive symptoms and 25-hydroxyvitamin D in middleaged and elderly Chinese. J Affect Disord 118, 240-243.

45. Engelberg H (1992) Low serum cholesterol and suicide. Lancet 339, 727-729.

46. Chrousos GP (2000) The role of stress and the hypothalamic-pituitary-adrenal axis in the pathogenesis of the metabolic syndrome: neuro-endocrine and target tissuerelated causes. Int J Obes Relat Metab Disord 24, Suppl. 2, S50-S55.

47. Delvin EE, Lambert M, Levy E et al. (2010) Vitamin D status is modestly associated with glycemia and indicators of lipid metabolism in French-Canadian children and adolescents. J Nutr 140, 987-991.

48. Gaddipati VC, Bailey BA, Kuriacose R et al. (2011) The relationship of vitamin D status to cardiovascular risk factors and amputation risk in veterans with peripheral arterial disease. J Am Med Dir Assoc 12, 58-61.

49. Dace A, Martin-el Yazidi C, Bonne J et al. (1997) Calcitriol is a positive effector of adipose differentiation in the OB 17 cell line: relationship with the adipogenic action of triiodothyronine. Biochem Biophys Res Commun 232, 771-776.

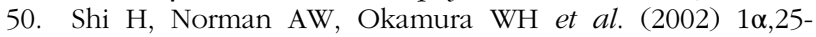
Dihydroxyvitamin $\mathrm{D}_{3}$ inhibits uncoupling protein 2 expression in human adipocytes. FASEB J 16, 1808-1810.

51. Welberg JW, Monkelbaan JF, de Vries EG et al. (1994) Effects of supplemental dietary calcium on quantitative and qualitative fecal fat excretion in man. Ann Nutr Metab 38, 185-191.

52. Christensen R, Lorenzen JK, Svith CR et al. (2009) Effect of calcium from dairy and dietary supplements on faecal fat excretion: a meta-analysis of randomized controlled trials. Obes Rev 10, 475-486.

53. Zemel MB, Shi H, Greer B et al. (2000) Regulation of adiposity by dietary calcium. FASEB J 14, 1132-1138.

54. Bogh MK, Schmedes AV, Philipsen PA et al. (2010) Vitamin D production after UVB exposure depends on baseline vitamin $\mathrm{D}$ and total cholesterol but not on skin pigmentation. J Invest Dermatol 130, 546-553.

55. Watkins CL, Lightbody CE, Sutton CJ et al. (2007) Evaluation of a single-item screening tool for depression after stroke: a cohort study. Clin Rehabil 21, 846-852.

56. Blozik E, Scherer M, Lacruz ME et al. (2013) Diagnostic utility of a one-item question to screen for depressive disorders: results from the KORA F3 study. BMC Fam Pract 14, 198. 